\title{
The association between deep learning approach and case based learning
}

Meenakshi Jhala* (D) and Jai Mathur

\begin{abstract}
Being medical students, and having experienced different learning approaches ourselves, here, we discuss and critically analyse the importance of the deep learning approach that Chonkar et al. have presented, alongside emphasizing Case Based Learning, and their roles in life long medical learning.
\end{abstract}

\section{Background}

We would like to thank Chonkar et al. [1] on their recent article which critically analyses the importance of different learning approaches and the long-term outcomes on retention of knowledge in medical students. Following on from the concept of the deep learning approach, we understand that it can be time consuming; hence, $50.8 \%$ [1] of students had the strategic approach as their dominant one. Chonkar et al. [1] have notably shown encouragement towards approaches which enhance the deeper learning approaches. In this letter, we would like to put emphasis on the case based learning method which transitions onto problem based learning after the two pre-clinical years at St George's University of London, alongside the outcome of the deep learning approach and different examinations.

\section{Main text}

As medical students ourselves, we have witnessed the evolution of pedagogical methods, and based upon personal reflection, have found that understanding a subject at its core is more valuable than a surface, superficial understanding. As aforementioned, at St George's, we have case based learning in the first 2 years of medical school which morphs into problem based learning from third year onwards. Several studies have shown the

\footnotetext{
* Correspondence: m1600868@sgul.ac.uk

St George's University of London, Cranmer Terrace, Tooting, London SW17 ORE, England, UK
}

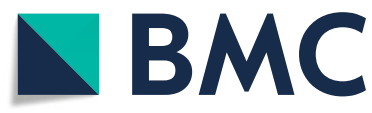

(c) The Author(s). 2019 Open Access This article is distributed under the terms of the Creative Commons Attribution 4.0 International License (http://creativecommons.org/licenses/by/4.0/), which permits unrestricted use, distribution, and reproduction in any medium, provided you give appropriate credit to the original author(s) and the source, provide a link to the Creative Commons license, and indicate if changes were made. The Creative Commons Public Domain Dedication waiver (http://creativecommons.org/publicdomain/zero/1.0/) applies to the data made available in this article, unless otherwise stated. by Mclean [2] believed that CBL "improves clinical performance and enhances clinical knowledge" which reinforces ours and Chonkar et al's points [1] of using case based learning as a technique to promote the deep approach [2]. Comparisons between problem based learning and case based learning have also been made, with CBL named more "goal-oriented" [3]. This is due to the input of the instructor which allows for each student to have an equal contribution towards the diagnosis of a patient; they are "content experts" for each CBL session. Moreover, "high self-regulated learners" display increased ease with case based learning [4] and in our opinion, this may be due to their already developed skills and techniques to efficiently complete independent tasks. Thus, we believe that should the deep learning approach be emphasized more, case based learning would have a more fruitful outcome in lifelong learning. We personally enjoy CBL due to the engagement and theory application; a review by Williams [5] gives evidence of this overall.

Having experienced CBL first hand, we can agree that wanting to culminate a session with determining the diagnosis of a patient, and applying knowledge acquired through lectures set in the curriculum to reach that step, derives a form of satisfaction. It also grants a glimpse of why the pre-clinical years are important; motivation can far too often become lost, when only learning theory for 2 years continuously, 
without a practical element, and links to the occupation of clinical practice. Having short placements alongside our pre-clinical years puts everything into an occupational perspective and allows us to clinically apply theoretical knowledge we have in understanding pathologies; this enriches and fortifies our knowledge. We feel that this could be another complementary or adjuvant way to encourage the deep learning approach Chonkar et al. [1] speak about, alongside case based learning.

Chonkar et al. [1] discuss the effect of education before medical school having an influence in the way students learn, and the approaches they take, at medical school. This discourse is understandable, since in pre-medical school education, there is a homogenous, universalized objective of getting into medical school, which is the foundation for why the strategic learning approach is emphasized so heavily, as it is about having the relevant qualifications to be able to be accepted onto such a competitive course. However, this alters significantly when in medical school, because it is no longer necessarily about achieving a certain percentage at all times, but rather about choosing between several different career trajectories. We have found, with personal experience, many students realize their learning approach is not allowing them to gain maximum knowledge until far too late. Students who are "average" may believe their methods of surface or strategic studying are working and stay at that level; many students may simply have the target of passing examinations, so if that is ascertained, the task has been completed. Therefore, we second the point that the impetus must be on universities to reinforce certain learning approaches in medical school, which instils deep strategic revision methods in students.

Focusing more on the deep learning approach, as we know, medical school prepares us for future practice as health care professionals. Therefore, it is important that the examinations test our knowledge; the challenge herein is that it is not possible to have a holistic view on the extent of knowledge obtained by students. This is especially evident when students only apply the strategic or surface approach and do not have the opportunity to apply their knowledge to practical situations, because that depth of knowledge has not been taught and reinforced. Ergo, we appreciate the importance of the Objective Structured Clinical Examination (OSCE) as that allows for application of knowledge - without prompts, which are given in multiple choice question examinations. This is because, as future practicing clinicians, in tangible, real life situations, there will be no 'hints' given, hence showing the value of the deep approach for life-long learning, to treat patients. It is, thus, imperative to highlight to students that they are studying to make monumental differences in the lives of patients, rather than just pass examinations.

As we proceed onto clinical years, we have witnessed first-hand, the significance of having a robust understanding of medical concepts, as these are our foundations for the next step of investigations and management in patient care. This can be especially difficult as many patients have non-specific presenting complaints, such as in the case of ovarian cancer, ultimately leaving it down to a thorough investigation and deep clinical knowledge base that will allow differentiation between two or three prime differentials. Thus, this can be a challenge; if the broad and basic knowledge of specialties taught in the pre-clinical years is not up to standard, this engenders an obstacle towards being a competent doctor by the end of our course. Therefore, we believe, it is in the clinical years where the surface/strategic approach shifts to the deep approach, as many students are made aware of the gaps in their knowledge, and strive to actively "read up" in order to ascertain the level of knowledge needed for true medical practice. Case based learning and problem based learning can allow opportunities for students to modify and transition their learning methods; however, it is on placements, where the practical scenarios are present, that students truly appreciate the need of understanding and applying the deep learning approach, rather than surface learning. In our opinion, the deep learning approach has a direct correlation between the competency of a future doctor being able to provide holistic care.

\section{Conclusion}

As the final aim is to treat patients, we believe case based learning is an outstanding start to transitioning students from more traditional and theoretical "text-book" methodologies, to practical scenarios. It allows students to think holistically, from the presenting complaint to the social aspect of the patient's life, and we believe this is due to the effective deep learning approach. The advantages of deep understanding can then be seen to amplify further in the clinical years. We believe that, if students applied the deep learning approach from when they started medical school, their knowledge would reach higher echelons, clinical problem-solving ability would be enhanced, and there would be less reliance on "memorization" of diseases, and more on understanding how symptoms can constellate to diagnoses. To conclude, we understand that students adapt their learning approaches with different tasks, however as Chonkar et al. [1] have mentioned in their study, the deep learning approach is most effective and most relevant to the practice of a Doctor. 


\author{
Response to "The association between deep learning approach and case based learning" \\ Kok Hian Tan 2,3,4, Sonali Prashant Chonkar ${ }^{2,3,4}$, Li Xian Amy Tan ${ }^{5}$ and Bernard Chern²,3,4,5 \\ ${ }^{2}$ KK Women's and Children's Hospital, Singapore, Singapore \\ ${ }^{3}$ Duke-NUS Medical School, Singapore, Singapore \\ ${ }^{4}$ Yong Loo Lin School of Medicine, National University of Singapore, Singapore, Singapore \\ ${ }^{5}$ Lee Kong Chian School of Medicine, Nanyang Technological University, Singapore, Singapore
}

We note that Jhala \& Mathur (both medical students) concurred with us on the importance of the deep learning approach for medical students. We agree that the Case Based Learning is useful for deep learning. However, due to time pressure and the format of knowledge and skills assessment, medical students often resort to superficial and strategic learning approaches to cope in medical schools.

Deep learning emphasizes understanding of concepts and relations, having an interest in ideas, application of knowledge and cognitively active learning behaviours [1]. Indeed, the essence of deep learning is real understanding and not memorisation of isolated facts. All medical schools need to play a part to ensure that the deep learning approach is rooted in their institutions. These involve evolving teaching and assessment methods to enhance deep learning for medical students. Assessment formats directly affect the choice of study strategies. If faculty are committed to preparing questions that require understanding and thinking skills, medical students will find that they cannot easily answer those questions with isolated facts that they have memorized. They will start learning differently. With better understanding, they can have better retention and effective application of the knowledge they acquired.

We are heartened that in many medical schools, pro-deep learning methods and assessments are increasingly utilised. These include Case Based Learning, Problem Based Learning, Team Based Learning and OSCE, which emphasise on understanding and applications in relation to the situation or scenario [6]. Forming a vibrant academic medicine culture with the healthcare system, where medical students can actively interact and learn from senior and also junior physicians in real life cases, is another excellent way for better teaching and deep learning [7].

It is important to note that learning methods should be holistic and multi-pronged. While basic facts and standard management to medical issues and problems can be managed through superficial and strategic learning approaches, real understanding with optimal retention of knowledge as well as optimal application appropriately customised to specific situations, require the deep learning approach. Not surprisingly, superficial learning assessment measures like non-scenario based multiple-choice questions, that test recall of facts, are increasingly being supplemented by pro-deep learning methods.

There is a need to enhance self-awareness of learning approaches so that the deep learning approach can be more consciously utilised as the predominant approach. Understanding of these learning approaches can be introduced in early medical student years to optimise learning for medical students.

Abbreviations

CBL: Case based learning; OSCE: Objective Structured Clinical Examination

Acknowledgements

None.

Funding

No funding was obtained for this manuscript.

Availability of data and materials

Not applicable.

Authors' contributions

MJ carried out the research of sources, insight, analysis and interpretation of data. JM carried out critical analysis and drafting of the manuscript. Both authors read and approved the final manuscript.

Ethics approval and consent to participate Not applicable.

Consent for publication

Not applicable.

Competing interests

The authors declare that they have no competing interests.

\section{Publisher's Note}

Springer Nature remains neutral with regard to jurisdictional claims in published maps and institutional affiliations.

Received: 22 August 2018 Accepted: 10 March 2019

Published online: 11 April 2019

References

1. Chonkar SP, et al. The predominant learning approaches of medical students. BMC Med Educ. 2018;18(17):1-8.

2. McLean S. Case-based learning and its application in medical and healthcare fields: a review of worldwide literature. J Med Educ Curric Dev. 2016;3: 39-49. https://doi.org/10.4137/JMECD.S20377.

3. Srinivasan $M$, et al. Comparing problem-based learning with case-based learning: effects of a major curricular shift at two institutions. Acad Med. 2007:82(1):74-82. https://doi.org/10.1097/01.ACM.0000249963.93776.aa. 
4. Ertmer $P$, Newby $T$, Macdougall M. Reflective self regulation as a facilitative factor in learning from case-based instruction. In: Proceedings of the 1995 Annual National Convention of the Association for Educational Communications and Technology. Anaheim: Institute of Education Sciences; 1995. p. 1-21.

5. Williams B. Case based learning - a review of the literature: is there scope for this educational paradigm in prehospital education? Emerg Med J. 2005; 22:577-81. https://doi.org/10.1136/emj.2004.022707.

6. Tan KH, Ng MJ, Tey WS, Tan HK, Chern B. Survey on academic medicine culture, enablers \& barriers in a newly formed academic department in Singapore. J Hosp Admin. 2016;5(5):30-40.

7. Kamei RK, Cook S, Janil P, Frank S. 21st Century learning in medicine: traditional teaching versus team-based learning. Med Sci Educ. 2012;22(2): $57-64$.

Ready to submit your research? Choose BMC and benefit from:

- fast, convenient online submission

- thorough peer review by experienced researchers in your field

- rapid publication on acceptance

- support for research data, including large and complex data types

- gold Open Access which fosters wider collaboration and increased citations

- maximum visibility for your research: over $100 \mathrm{M}$ website views per year

At $\mathrm{BMC}$, research is always in progress.

Learn more biomedcentral.com/submissions 